\title{
Fishing out the signal in polypharmacological high-throughput screening data using novel navigator cheminformatics software
}

\author{
Denis Fourches', Alexander Tropsha \\ From 9th German Conference on Chemoinformatics \\ Fulda, Germany. 10-12 November 2013
}

Many drugs are characterized by polypharmacological mechanisms of action. Thus, prospective drug discovery studies often start by testing large compound libraries in multiple and diverse High-Throughput Screening (HTS) assays. These large heterogeneous data collections pose numerous computational challenges concerning processing, curation, and analysis of untreated output files generated by plate readers. We have developed the freely-accessible HTS Navigator software to enable and facilitate the processing and analysis of polypharmacological HTS data. We report on the capabilities of Navigator and present several case studies where we employed cheminformatics approaches embedded within the Navigator to curate and analyze large datasets of compounds tested toward different panels of targets. Examples include libraries of compounds tested for their inhibition potencies across several CYP450; or for their inhibition of multiple protein kinases; or their binding profiles against multiple GPCRs. We show how to quickly identify and highlight compounds with unique mono- and dual- selectivity for certain targets in the curated HTS matrix. We discuss the problem of experimental variability in HTS data and its consequences for molecular modeling and emphasize the synergistic potential of different cheminformatics approaches to detect both false-positive and false-negative compounds using neighborhood analysis and target baseline correction factors. Finally, we describe the Chemical-Biological ReadAcross (CBRA) approach [1] also implemented in the Navigator to infer the activity of external compounds from both chemical (defined by chemical similarity) and

Laboratory for Molecular Modeling, Division of Chemical Biology and Medicinal Chemistry, UNC Eshelman School of Pharmacy, University of North Carolina at Chapel Hill, Chapel Hill, NC, 27599, USA biological (defined by the similarity of HTS profiles) analogues.

Published: 11 March 2014

Reference

1. Low Y, Sedykh A, Fourches D, Golbraikh A, Whelan M, Rusyn I, Tropsha A: Integrative Chemical-Biological Read-Across Approach for Chemical Hazard Classification. Chem Res Toxicol 2013.

doi:10.1186/1758-2946-6-S1-P14

Cite this article as: Fourches and Tropsha: Fishing out the signal in polypharmacological high-throughput screening data using novel navigator cheminformatics software. Journal of Cheminformatics 2014 6(Suppl 1):P14.

\section{Publish with ChemistryCentral and every scientist can read your work free of charge \\ "Open access provides opportunities to our colleagues in other parts of the globe, by allowing anyone to view the content free of charge." \\ W. Jeffery Hurst, The Hershey Company. \\ - available free of charge to the entire scientific community \\ - peer reviewed and published immediately upon acceptance \\ - cited in PubMed and archived on PubMed Central \\ - yours - you keep the copyright \\ Submit your manuscript here: \\ http://www.chemistrycentral.com/manuscript/}

